OPEN ACCESS

Edited by:

Hector Mora Montes,

University of Guanajuato, Mexico

Reviewed by:

Delia Vanessa Lopez Guerrero, Universidad Autónoma del Estado de

Morelos, Mexico

Joseph M. Bliss,

Women \& Infants Hospital of Rhode Island, United States

*Correspondence:

Claudia Monari claudia.monari@unipg.it

Specialty section: This article was submitted to Fungi and Their Interactions, a section of the journal Frontiers in Microbiology

Received: 08 April 2019 Accepted: 12 June 2019

Published: 11 July 2019

Citation

Roselletti E, Sabbatini S, Ballet N, Perito S, Pericolini E, Blasi E, Mosci P, Cayzeele Decherf A, Monari $C$ and Vecchiarelli A (2019) Saccharomyces cerevisiae CNCM I-3856 as a

New Therapeutic Agent Against Oropharyngeal Candidiasis.

Front. Microbiol. 10:1469. doi: 10.3389/fmicb.2019.01469

\section{Saccharomyces cerevisiae CNCM I-3856 as a New Therapeutic Agent Against Oropharyngeal Candidiasis}

\author{
Elena Roselletti', Samuele Sabbatini', Nathalie Ballet ${ }^{2}$, Stefano Perito', Eva Pericolini ${ }^{3}$, \\ Elisabetta Blasi ${ }^{3}$, Paolo Mosci ${ }^{4}$, Amélie Cayzeele Decherf ${ }^{5}$, Claudia Monari ${ }^{\text {P* }}$ and \\ Anna Vecchiarelli'
}

\begin{abstract}
${ }^{1}$ Medical Microbiology Section, Department of Medicine, University of Perugia, Perugia, Italy, ${ }^{2}$ Lesaffre International, Lesaffre Group, Marcq-en-Baroeul, France, ${ }^{3}$ Department of Surgical, Medical, Dental and Morphological Sciences With Interest in Transplant, Oncological and Regenerative Medicine, University of Modena and Reggio Emilia, Modena, Italy, ${ }^{4}$ nternal Medicine Section, Department of Veterinary Medicine, University of Perugia, Perugia, Italy, ${ }^{5}$ Gnosis by Lesaffre, Lesaffre Group, Marcq-en-Baroeul, France
\end{abstract}

Oropharyngeal candidiasis is a common opportunistic mucosal infection of the oral cavity, mainly caused by an overgrowth of Candida albicans. This infection can inhibit nutritional intakes and strongly affect quality of life. To date, standard therapeutic strategies involving the administration of antifungal drugs can bring several side effects, not least the emergence of drug-resistant strains. The purpose of this study is to investigate the effectiveness of Saccharomyces cerevisiae CNCM I-3856 (live or inactivated cells) against oropharyngeal candidiasis. Our results show that administration of $S$. cerevisiae CNCM I-3856 (live or inactivated cells) in the oral cavity of C57BL/6J mice resulted in a protective effect against oropharyngeal candidiasis. The strongest effect was obtained with live S. cerevisiae CNCM I-3856. This was related to: (1) a decrease in C. albicans load in the oral cavity, esophagus, stomach, and duodenum; (2) an early resolution of inflammatory process in the tongue; (3) a marked reduction in C. albicans virulence factors; and (4) a consistent increase in neutrophil antimicrobial capacity. These findings suggest that $S$. cerevisiae products are potentially beneficial in the treatment of oropharyngeal candidiasis.

Keywords: oropharyngeal candidiasis, oral infections, probiotics, Saccharomyces cerevisiae, Candida albicans, yeasts

\section{INTRODUCTION}

Candida albicans is an opportunistic pathogenic fungus that commonly inhabits the mouth, vagina, and intestinal tract of healthy individuals, causing many different types of mucosal infections, including oral candidiasis. Also known as thrush or oropharyngeal candidiasis (OPC), oral candidiasis is the most common opportunistic infection of the oral cavity (Akpan and Morgan, 2002; Gonzalez Gravina et al., 2007; Naglik and Moyes, 2011; Melkoumov et al., 2013; Berberi et al., 2015; Kenno et al., 2016). It is especially common and underdiagnosed among the elderly (particularly in those wearing dentures), infants, and immunocompromised individuals or individuals on long-term antibiotic treatments. It can also be linked to systemic diseases such as diabetes mellitus. In individuals with defective immune response, 
the pathogen may spread to the pharynx and the esophagus, causing severe symptoms such as erosions and ulcerations of the tissues. In addition, it spreads through the gastrointestinal tract, predisposing its host to the development of systemic or disseminated candidiasis, leading to high morbidity and mortality rates (Gudlaugsson et al., 2003; Pappas, 2006). Indeed, compelling evidence shows that the gastrointestinal tract could be the largest source of candidemia when systemic or local mucosal immune functions are disturbed (Li et al., 2000; Miranda et al., 2009; Schulze and Sonnenborn, 2009). To date, antifungal drug administration (nystatin, amphotericin $\mathrm{B}$, fluconazole (FLZ), itraconazole, and voriconazole) represents the first line therapy against candidiasis (Garcia-Cuesta et al., 2014). However, the numerous side effects (Ohshima et al., 2016) induced by drugs toxicity (Sardi et al., 2013) and the appearance of drug-resistant strains (Matsubara et al., 2016; Ohshima et al., 2016) emphasize the urgency of developing innovative therapeutic strategies. In this regard, probiotics can represent a promising alternative approach. Several studies have reported the anti-pathogenic potential of probiotic bacteria for the prevention and/or the treatment of oropharyngeal candidiasis (Hatakka et al., 2007; Ishijima et al., 2012; Ishikawa et al., 2015; Kraft-Bodi et al., 2015; Matsubara et al., 2016; Ohshima et al., 2016). Recently, Leão et al. (2018) observed that the presence of Lactobacillus rhamnosus ATCC 7469, prior to inoculation with C. albicans clinical strain, avoided the colonization and consequently the growth of the pathogen, thus effectively preventing the development of candidiasis in immunosuppressed mice. To our knowledge, only one study supported the efficacy of Saccharomyces cerevisiae treatment by local application (Premanathan et al., 2011). Unfortunately, the S. cerevisiae strain used in this study was not defined. We recently demonstrated that live and inactivated S. cerevisiae CNCM I-3856 show a therapeutic activity in an experimental model of vaginal candidiasis in mice (Pericolini et al., 2017; Gabrielli et al., 2018). This beneficial effect was induced by $S$. cerevisiae co-aggregation with $C$. albicans, decrease of $C$. albicans adherence to epithelial cells, and inhibition of some important virulence factors such as the pathogen's ability to switch from bud to hyphal form. In this study, we test the effectiveness of live and inactivated S. cerevisiae CNCM I-3856 against OPC and examine the mechanisms of action allowing this beneficial effect.

\section{MATERIALS AND METHODS}

\section{C. albicans Strain and Culture}

C. albicans CA1398 carrying the ACT1p-gLUC59 fusion (gLUC59) was used (Enjalbert et al., 2009). The gLUC59 luciferase reporter has previously been described (Enjalbert et al., 2009). C. albicans gLUC59 (BLI-Candida) was cultured in yeast peptone dextrose (YPD) as described by Solis and Filler (2012). The C. albicans CA 1398 carrying the ACT1pgLUC59 fusion and its parental strain CA 1938 were equally pathogenic as previously demonstrated (Enjalbert et al., 2009).

\section{Study Products}

The products studied were provided by Gnosis by Lesaffre (Marcq-en-Baroeul, France). S. cerevisiae live yeast (referenced as GI) is a proprietary, well-characterized strain of Lesaffre, registered in the French National Collection of Cultures of Microorganisms (CNCM) under the number I-3856. The $S$. cerevisiae species is characterized by using phenotypic (API ${ }^{\circledR} \mathrm{ID} 32 \mathrm{C}$, Biomerieux SAS) and genotypic referenced methods (genetic amplification and sequencing of 26S DNA; Kurtzman and Robnett, 1997, 1998). Moreover, the strain CNCM I-3856 has been characterized by polymerase chain reaction (PCR) Interdelta typing techniques (European Committee for Standardization, 2009), and its genome has been sequenced. The inactivated yeast $S$. cerevisiae CNCM I-3856 (referenced as IY) is a primary grown dried whole yeast, obtained by drum drying of S. cerevisiae CNCM I-3856 and inactivated through the process of drying. Furthermore, the strain of L. rhamnosus GG (referenced as G 250) is registered in the American Type Culture Collection (ATCC) under the number 53103. The CFU counts of the probiotics used in these experiments are at least of $5 \times 10^{9} \mathrm{CFU} / \mathrm{g}$ for GI and $2.5 \times 10^{11} \mathrm{CFU} / \mathrm{g}$ for $\mathrm{G} 250$. All products were used at $100 \mathrm{mg} / \mathrm{ml}$.

\section{Mouse Model of Oropharyngeal Candidiasis}

Female C57BL/6J mice from Charles River (Calco, Italy) were used at 6-8 weeks of age. Mice were treated subcutaneously with $225 \mathrm{mg} / \mathrm{kg}$ cortisone acetate (Sigma-Aldrich) every 2 days starting from 1 day before infection until the end of experiment. After anesthesia with a subcutaneous injection of a mixture of Tiletamine/Zolazepam-Xylazine (50-5 mg/kg) (Mosci et al., 2013) mice were infected with $1 \times 10^{6} \mathrm{CFU} / \mathrm{ml}$ BLI-Candida suspension as previously described (Solis and Filler, 2012).

Then mice were treated sublingually with $10 \mu \mathrm{l}$ of saline, FLZ (4 mg/ml), GI, IY, or G 250 (all $100 \mathrm{mg} / \mathrm{ml}$ ) on days $+1,+2,+3$, and +6 post-infection. The oral cavity was swabbed just before the infection and streaked on YPD agar plus chloramphenicol ( $50 \mu \mathrm{g} / \mathrm{ml}$; both from Sigma-Aldrich) to verify the absence of Candida spp.

\section{Real-Time Monitoring of Oropharyngeal Candidiasis and Pathogen Burden Determination}

Starting on day 1 after challenge and at each selected day, $10 \mu \mathrm{l}\left(0.5 \mathrm{mg} / \mathrm{ml}\right.$ in $1: 10$ methanol: $\left.\mathrm{H}_{2} \mathrm{O}\right)$ of coelenterazine (Synchem, OHM) was added sublingually. Mice were then imaged in the IVIS Lumina XRMS Imaging system (Perkin Elmer) under subcutaneous anesthesia. The total photon emission was quantified as previously described (Mosci et al., 2013). In the selected experiments, an ex vivo analysis of esophagus, stomach, kidneys, liver, and feces from OPC mice was performed at day +8 post-infection as previously described (Mosci et al., 2014). The C. albicans burden of the tongue, esophagus, stomach, and duodenum was evaluated at days $+3,+6$ and +8 post-infection as previously described (Mosci et al., 2014). 


\section{Histological Analysis}

The animals infected and treated with saline, FLZ, IY, GI, or G 250 were sacrificed at day +8 post-infection to analyze gross and histopathologic lesions, and tongues were excised. Histological examination was, also, performed on tongues of uninfected mice treated with saline, FLZ, GI, IY, or G 250 to evaluate the integrity of the tissue and neutrophil recruitment. The tissues were fixed immediately in $10 \%$ formalin and then embedded in paraffin. The tongues were sectioned longitudinally to evaluate the extension of the lesions. The 3-5 $\mu \mathrm{m}$ thick sections were stained using the periodic acid-Schiff (PAS) procedure to detect fungi and examined by light microscopy (Leica DM2500). The scale bars are in micrometer.

\section{Quantitative Analysis of ALS3, SAP2, and SAP6 Gene Expression}

At day +6 post-infection tongue homogenates from mice infected and treated with saline, FLZ, GI, or IY were centrifuged at 3,000 rpm for $5 \mathrm{~min}$. Then cellular fractions were lysed using Trizol reagent (Life Technology). Total RNA was extracted and retro-transcribed by using the Moloney murine leukemia virus reverse transcriptase reaction (M-MLV RT) as described in the manufacturer's instructions. cDNA concentration was determined using a spectrophotometer. C. albicans ACT1, SAP2, SAP6, and ALS3 gene transcription was detected by using primers reported in the literature (Naglik et al., 2008; Roudbarmohammadi et al., 2016). Real-time quantitative PCR (RT-qPCR) was performed in 96-well PCR SYBR green plates (all from BioRad) using $200 \mathrm{ng}$ of cDNA for each sample. In this way, the amount of genes expressed by $C$. albicans gLUC59 during infection was independent by fungal burden. All samples were measured in triplicate. The relative level of Candida gene expression was reported as $2-\Delta \Delta \mathrm{Ct}$ relative to transcripts of $C$. albicans inoculum (basal gene expression level of Candida at day 0). Briefly, 3 days before infection, a colony of the C. albicans gLUC59 was added into $10 \mathrm{ml}$ of YPD broth and incubate in a $30^{\circ} \mathrm{C}$ shaker. The next day, $100 \mu \mathrm{l}$ of the overnight culture were transferred to $10 \mathrm{ml}$ fresh YPD broth and incubate in a $30^{\circ} \mathrm{C}$ shaker overnight. This step was repeated one more time. The next day, C. albicans gLUC59 was recovered, counted, and diluted at desired concentration (Solis and Filler, 2012). Amplification conditions used were the same for ACT1, ALS3, SAP2, and SAP6 genes: $3 \mathrm{~min}$ at $95^{\circ} \mathrm{C}, 40$ cycles of $10 \mathrm{~s}$ at $95^{\circ} \mathrm{C}$ and $30 \mathrm{~s}$ at primer specific annealing temperature. The experiments were performed using the Eppendorf Master cycler.

\section{Candidacidal Assays}

At day +6 post-infection, peritoneal neutrophils of mice orally infected and treated as described in section "Materials and Methods" with saline, FLZ (4 mg/ml), GI, or IY (both at $100 \mathrm{mg} / \mathrm{ml}$ ) were collected $18 \mathrm{~h}$ after the intraperitoneal injection of $0.5 \mathrm{ml}$ endotoxin-free $10 \%$ thioglycolate solution (Difco) The percentage of neutrophils was $>90 \%$ (Mosci et al., 2014). The oxidative burst of neutrophils was detected by labeling cells $\left(4 \times 10^{6} / \mathrm{ml}\right)$ with $1 \mu \mathrm{M}$ of $2^{\prime}, 7^{\prime}$-dichlorofluoresceindiacetate (DCFH-DA) for $30 \mathrm{~min}$ at room temperature. Cells were then incubated with $C$. albicans (CA-6) $\left(2 \times 10^{6} \mathrm{CFU} / \mathrm{ml}\right)$ into a black 96-well plate (Nunc), and the emission of fluorescence was measured as previously described (Mosci et al., 2014; Ricci et al., 2019). Quantification of reactive oxygen species (ROS) production was determined by calculation of the area under curve (AUC) (Ermert et al., 2009; Kenno et al., 2016). Killing activity of neutrophils was determined by CFU assays. Briefly $0.1 \mathrm{ml} /$ well of neutrophils $\left(10^{5}\right.$ cells) was immediately incubated in flat-bottom 96 -well microtiter tissue culture plate with $0.1 \mathrm{ml} /$ well $\left(10^{4}\right.$ cells $)$ of C. albicans (CA-6) in RPMI-1640 plus 5\% FCS for $2 \mathrm{~h}$ at $37^{\circ} \mathrm{C}$ plus $5 \% \mathrm{CO}_{2}$. After incubation, plates were vigorously shaken, and cells were lysed by adding Triton X-100 (0.1\% in distilled water; final concentration in the well was $0.01 \%$ ). Serial dilutions were prepared in distilled water for each well. The samples were then spread on Sabouraud dextrose agar plus chloramphenicol $(50 \mu \mathrm{g} / \mathrm{ml})$ in triplicate, and CFU counts were determined after $24 \mathrm{~h}$ of incubation at $37^{\circ} \mathrm{C}$. Control cultures consisted of C. albicans (CA-6) incubated in RPMI-1640 plus 5\% FCS without effector cells. Killing activity was expressed as the percentage of CFU reduction with respect to neutrophils from uninfected mice and according to the following formula: \% killing activity $=100-(\mathrm{CFU}$ experimental/CFU control) $\times 100$.

\section{Statistical Analysis}

GraphPad Prism 7.0 software was used for all statistical analysis presented. Data are reported as boxplot graphics with median and the 25th and 75th percentile or mean \pm SEM from the experiments indicated in each figure legend. Statistical analysis was performed using Mann-Whitney $U$ test. $p<0.05$ was considered as significant.

\section{RESULTS}

\section{Effect of S. cerevisiae Treatment on Oropharyngeal Candidiasis}

To analyze whether inactivated $S$. cerevisiae (IY), live S. cerevisiae (GI), or live L. rhamnosus GG (G 250) were able to affect the course of oropharyngeal candidiasis, C57BL/6J mice were treated subcutaneously with cortisone acetate every 2 days starting from 1 day before infection until the end of the experiment. Briefly, the mice were infected with bioluminescent (BLI) C. albicans $\left(1 \times 10^{6} \mathrm{CFU} / \mathrm{ml} ; 10 \mu \mathrm{l} /\right.$ mouse $)$ as previously described by Solis and Filler (2012). The animals were then treated sublingually with saline, FLZ ( $4 \mathrm{mg} / \mathrm{ml} ; 10 \mu \mathrm{l} /$ mouse), IY, GI, or G 250 (all $100 \mathrm{mg} / \mathrm{ml} ; 10 \mu \mathrm{l} /$ mouse) on days +1 , $+2,+3$, and +6 post-infection. Saline-treated and FLZ-treated infected mice were used as negative and positive controls, respectively. The effect of the various treatments on oral candidiasis was evaluated at days $+1,+3,+6$, and +8 postinfection by measurement of photon emission from the oral cavity as well as by determination of CFU counts on the tongue. As shown in Figure 1, both IY and GI significantly 
decreased the BLI-C. albicans load in the oral cavity at each time point tested, both as visually evident (Figure 1A) and as measured by total photon emission (Figure 1B). Of note, the anti-Candida effect of IY and GI was comparable to the effect of positive control FLZ at each time point tested. By contrast, no effect was observed for G 250 (Figure 1B). The reduction of the Candida load in the oral cavity was also confirmed through a significant decrease in CFU counts on the tongues of IY- and GI-treated infected animals (Figure 2A). In order to characterize the lesions associated with the OPC, we performed histological analysis of the tongues at day +8 post-infection. The fungal invasion of the tongue of salinetreated infected mice was limited to a portion on the surface of the tongue due to the formation of pseudomembranes. The pathogenic fungi and the inflammatory cells appeared to be confined to the keratinized layer of the tongue where micro abscesses formation could be observed. No Candida cells and Candida-induced lesions were found in the tongues of FLZ, IY, and GI-treated infected mice (Figure 2B), while Candidainduced lesions were observed in the tongue of G 250-treated infected mice (Figure 2B). Histological examination of the tongues of uninfected mice treated with saline, FLZ, IY, GI, or G 250 showed complete tissue integrity and an absence of neutrophils recruitment (Figure 2B).

The progression of the infection to esophagus, stomach, and intestine was evaluated by CFU counts at days $+3,+6$, and +8 post-infection. The results show that both IY and GI were able to reduce the esophageal Candida load only at day +8 post-infection (Figure 3A). In the stomach, the beneficial effect of GI was evident at both days +6 and +8 post-infection, while the effect of IY was appreciable only at day +8 post-infection (Figure 3B). In the duodenum, only GI induced a significant inhibition of the $C$. albicans load at days +6 and +8 postinfection (Figure 3C). By contrast, G 250 had no significant effect (Figure 3). Notably and as expected, FLZ was active (Figure 3). Given that the treatment with the Lactobacillus probiotic did not influence the course of oropharyngeal infection, only $S$. cerevisiae products were evaluated in the subsequent experiments. First, the effect of IY and GI was evaluated by ex vivo bioluminescence emission of explanted gastric tracts (pharynx, esophagus, and stomach) at day +8 post-infection. The results show that treatment with IY and GI strongly reduced the BLI signal from the explanted organs as compared to organs from saline-treated infected mice (Figure 4). Next, the BLI signal from feces was tested to examine whether IY and GI were able to accelerate the passage of $C$. albicans from the stomach to intestine. The results show that BLI-C. albicans was evident only in the feces of saline-treated infected mice (Figure 4). Fungus dissemination to kidneys and liver was also evaluated. No fungal load was detected in these organs in any experimental group at day +8 post-infection (Figure 4). Further observations show that, by day +8 post-infection, infected mice did not show relevant clinical signs and manifested varying extents of weight loss. G 250- and saline-treated infected mice both showed similar extent of weight loss, while the weight loss in IY- and GY- treated groups was much less pronounced (data not shown).

\section{Effect of S. cerevisiae Treatment on Hyphae-Associated Virulence Genes}

In complementary experiments, we wondered if the inhibition of the pathogen load in the oral cavity may be related to the modulation of some important $C$. albicans virulence factors involved in the adhesion and invasion of epithelial cells (Naglik et al., 2011). To this end, the expression of ALS3 and two aspartyl proteases (SAP2 and 6) were analyzed. As shown in Figure 5A, the expression of the three virulence genes was significantly downregulated by the treatment with GI, but not with IY.

\section{Effect of S. cerevisiae Treatment on Neutrophil Activity}

Neutrophils represent a major class of immune effector cells in fungal killing (Gazendam et al., 2016), of which reactive oxygen species production is the major killing mechanism (Moyes and Naglik, 2011), and they play a central role in protecting oral epithelium from C. albicans injury (Weindl et al., 2007). Given the difficulty to recover these cells from mucosal tissue, we examined whether the neutrophils recruited in the peritoneal cavity had candidacidal activity. The results reported in Figure 5B show that neutrophils from infected mice presented a reduction in killing activity as compared to those from uninfected mice and that the in vivo treatment with GI resulted in a greater increase in candidacidal activity of neutrophils compared to that of the two other treated groups. Moreover, the oxidative burst of neutrophils was also increased following the GI treatment. As expected, FLZ was able to increase antimicrobial capacity of neutrophils (Brummer and Stevens, 1996; Figure 5B).

\section{DISCUSSION}

Probiotics are defined as live microorganisms, which, when administered in appropriate amount, provide a health benefit to the host (Food and Agriculture Organization and World Health Organization Expert Consultation, 2001). The numerous mechanisms by which probiotics accomplish their beneficial actions include competitive exclusion for binding sites of pathogenic microorganisms, production of anti-microbial substances, enhancement of the epithelial barrier, and modulation of the immune system. We recently demonstrated a clear effect of some probiotics in improving the course of mucosal infections in experimental models. In particular, daily treatment with S. cerevisiae CNCM I-3856 resulted in curing bacterial vaginosis (Pericolini et al., 2017; Sabbatini et al., 2018) and vaginal candidiasis in infected mice (Gabrielli et al., 2018). Here we demonstrated that the administration of live (GI) or inactivated (IY) S. cerevisiae CNCM I-3856 in the oral cavity results in a protective effect against oral candidiasis. A complementary set of experiments allows us to demonstrate that this protective effect is related to: (1) a significant inhibition of the C. albicans load in the oral cavity as well as in the tongue that showed an early resolution of the inflammatory process and (2) a progressive reduction of the pathogen load measured in the esophagus, stomach, and duodenum resulting in a clearance 
A
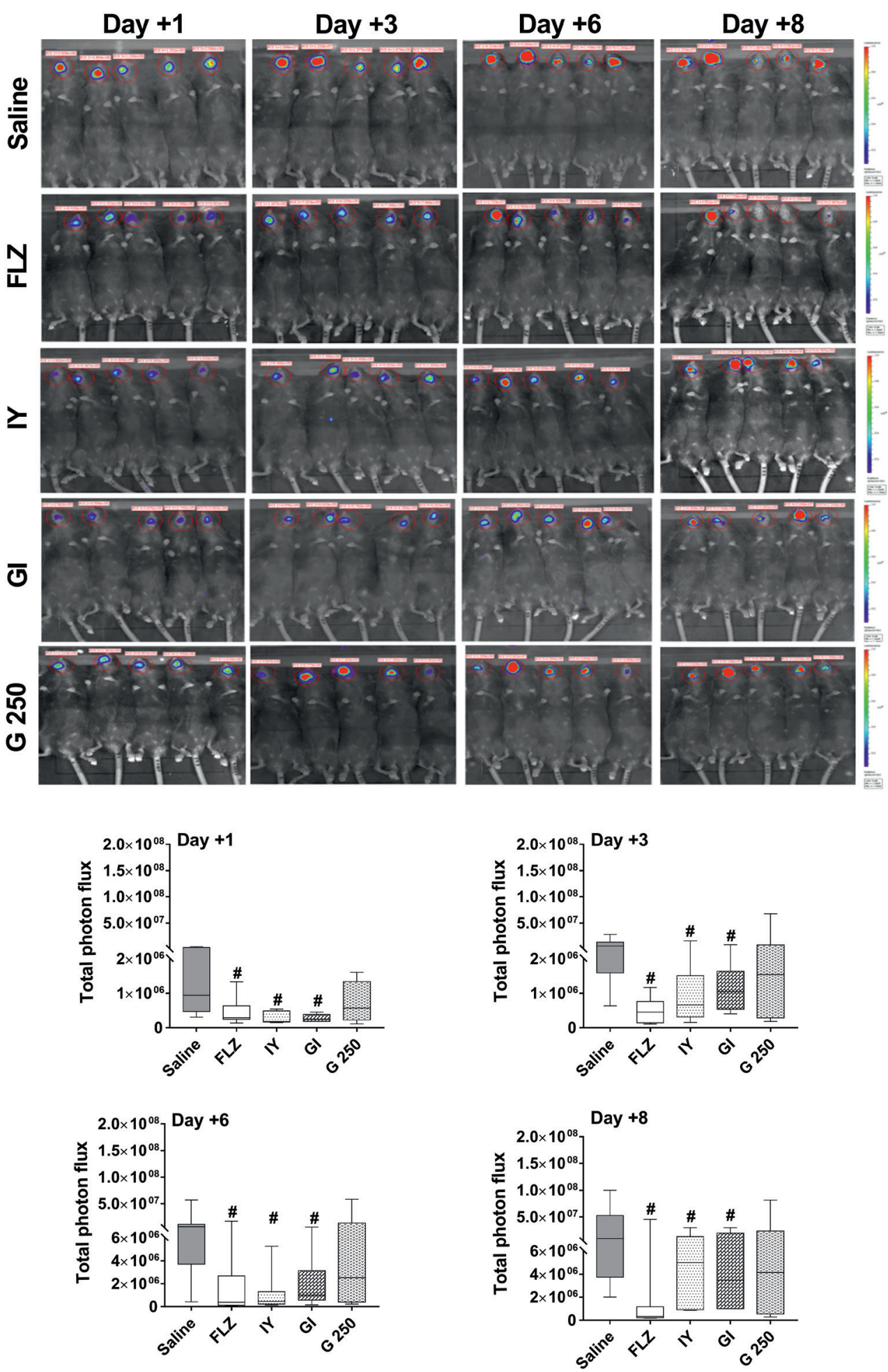

FIGURE 1 | In vivo imaging of mice orally infected with BLI-C. albicans and treated with FLZ, IY, Gl, or G 250. Mice were infected with BLI-C. albicans $\left(1 \times 10^{6} \mathrm{CFU} / \mathrm{ml}\right)$ and treated sublingually with $10 \mu \mathrm{l}$ of saline, FLZ (4 mg/ml), IY, Gl, or G 250 (all $\left.100 \mathrm{mg} / \mathrm{ml}\right)$. At 1, 3, 6, and 8 days post-infection anesthetized mice were treated sublingually with $10 \mu \mathrm{l}$ of coelenterazine $(0.5 \mathrm{mg} / \mathrm{ml})$ and imaged in the Lumina XRMS Imaging system. Images are representative of two separate experiments with similar results. (A) Total photon flux emission from oral within the images (Region Of Interest, ROI) of each mouse was quantified with Living Image R software package. (B) Quantification of Total photon flux emission from ROI. Data are from two different experiments each with $n=5$ mice/group. The boxplot graphics show median including the 25th and 75 th percentile. $" p<0.05$ FLZ or compound-treated infected mice vs. saline-treated infected mice. 


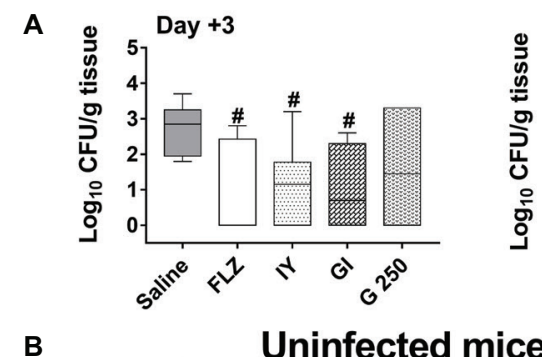

B

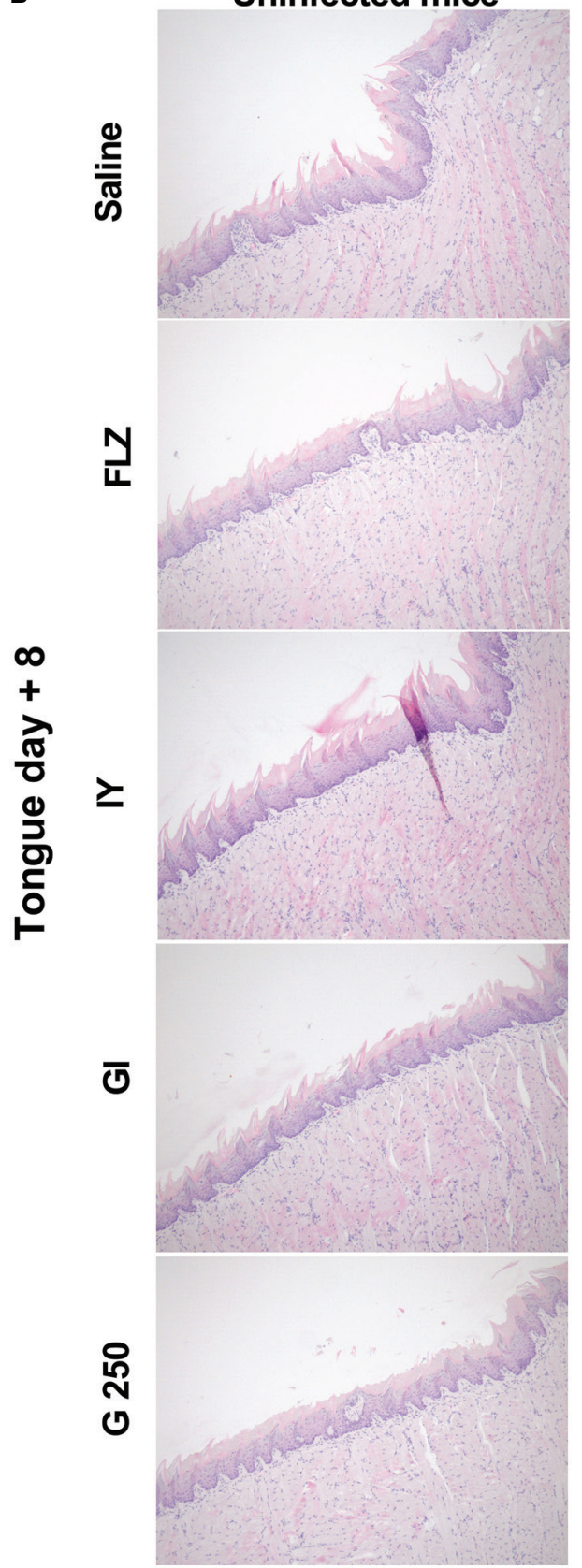

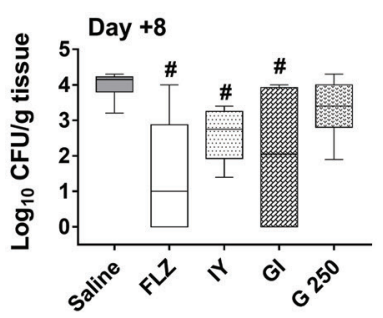

Infected mice

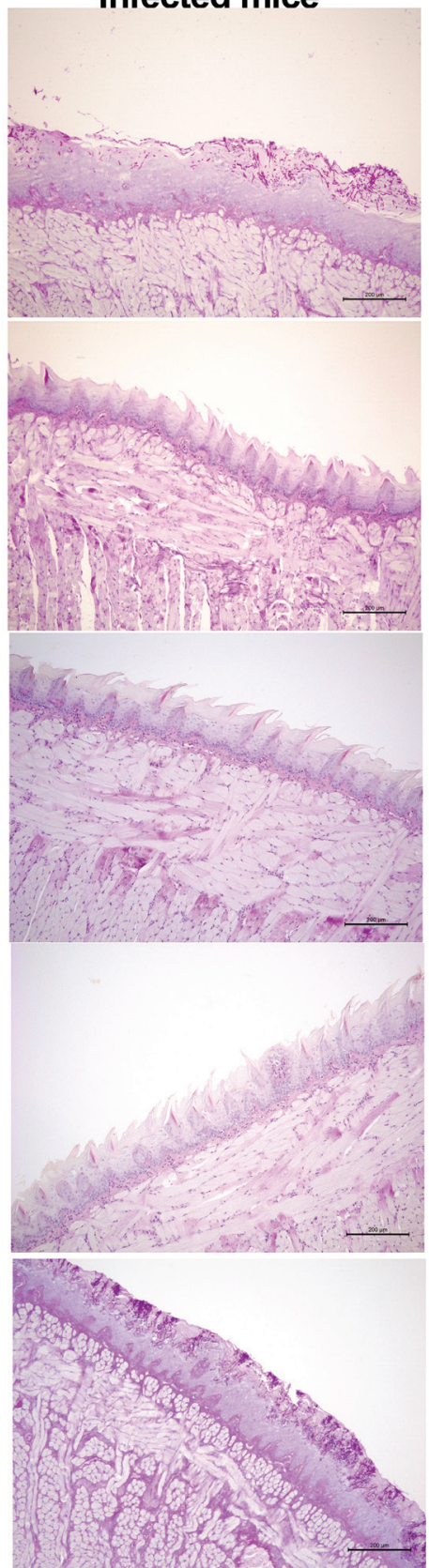

FIGURE 2 | Effect of IY, GI, or G 250 treatment on tongue fungal burden and histological inflammation. (A) Fungal burden of mice treated with 10 $\mu$ l of saline, FL Z ( $4 \mathrm{mg} / \mathrm{ml}$ ), IY, Gl, or G 250 (all $100 \mathrm{mg} / \mathrm{ml}$ ) was evaluated by CFU count at days $+3,+6$, and +8 post-infection in tongue of infected mice. Data are from two different experiments each with $n=3$ mice/group. The boxplot graphics show median including the 25th and 75 th percentile. ${ }^{*} p<0.05 \mathrm{FLZ}$ or compound-treated infected mice vs. saline-treated infected mice. (B) Tongue sections from uninfected and infected mice treated with $10 \mu \mathrm{l}$ of saline, FLZ (4 mg/ml), IY, GI, and G 250 (all $100 \mathrm{mg} / \mathrm{ml}$ ) are shown (day +8 post-infection). Images (Bar $=200 \mu \mathrm{m}$, magnification $10 x$ ) are representative of two separate experiments with similar results. 


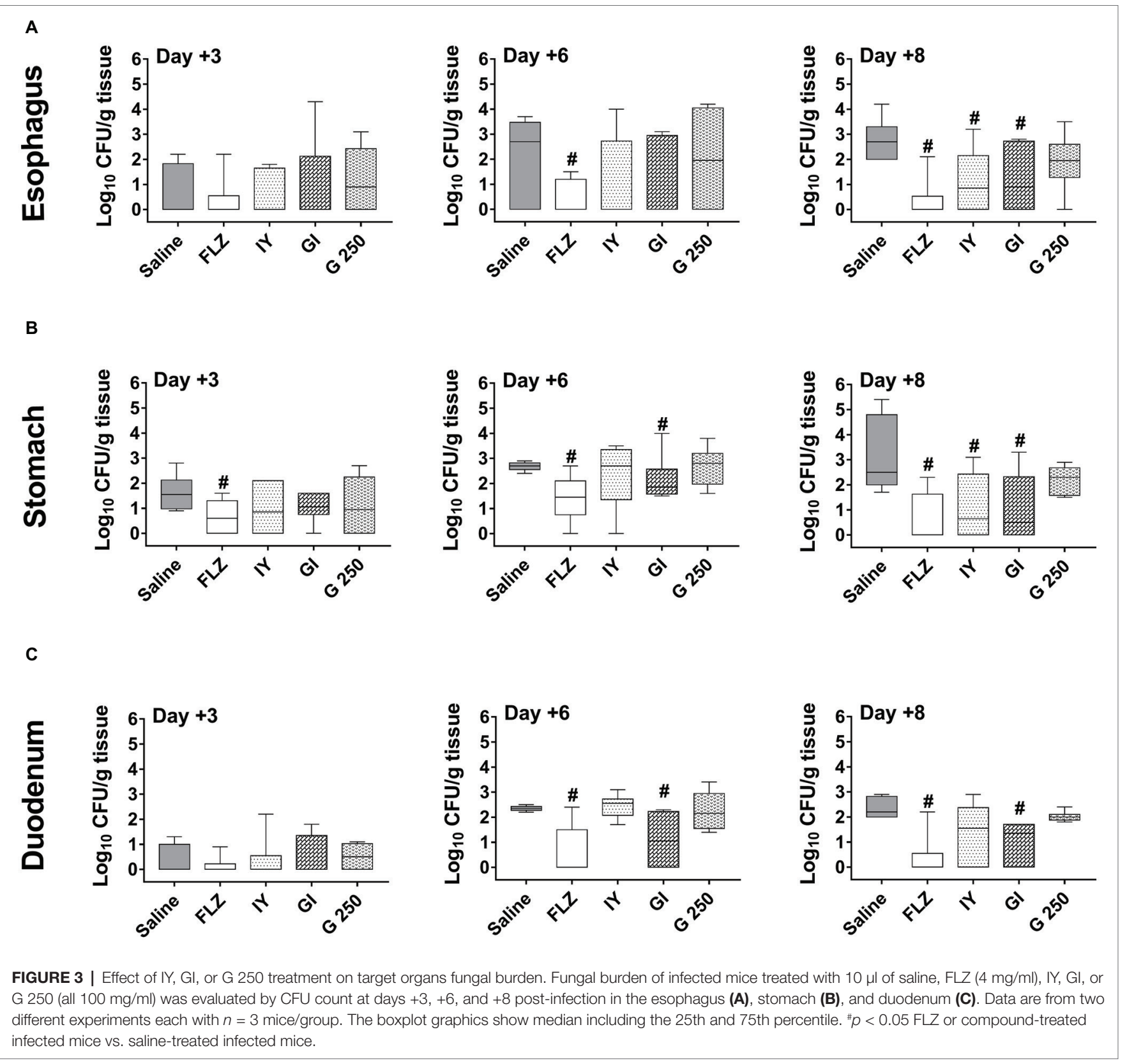

of C. albicans from the intestine. The marked increase in the antimicrobial capacity of neutrophils was related to GI upregulation of oxygen-dependent mechanisms. GI was, also, able to inhibit the expression of some Candida virulence factors, which is not verified for IY. Although the two yeast products have shown beneficial effects, these appeared to be more significant with live yeast. This is consistent with previous data showing that viable bacteria are more effective than non-viable bacteria for health benefit (Lahtinen, 2012).

Previous studies demonstrate that L. rhamnosus is able to interfere with $C$. albicans growth in in vitro experimental model (Allonsius et al., 2017). Our data show that L. rhamnosus GG was ineffective in our in vivo model of oral candidiasis, and this apparent discrepancy may be due to different experimental models used. Indeed, the effect of $L$. rhamnosus against candidiasis is hitherto unsubstantiated with in vivo evidence.

Despite the growing favor for the use of probiotics for oral infections (Cagetti et al., 2013; Laleman et al., 2014; Gruner et al., 2016), only few studies focusing on probiotic bacteria (lactobacilli and bifidobacteria) are available. These studies target specifically the elderly (Ai et al., 2017). None of the studies investigated yeast strains.

In this study, and for the first time, we provide evidence for the efficacy of a probiotic yeast in accelerating the clearance of C. albicans in an experimental model of OPC in mice. Furthermore, the daily administration of $S$. cerevisiae CNCM I-3856 resulted in a drastic reduction of pathogenic fungal load that is also related to the early quenching of the inflammatory 


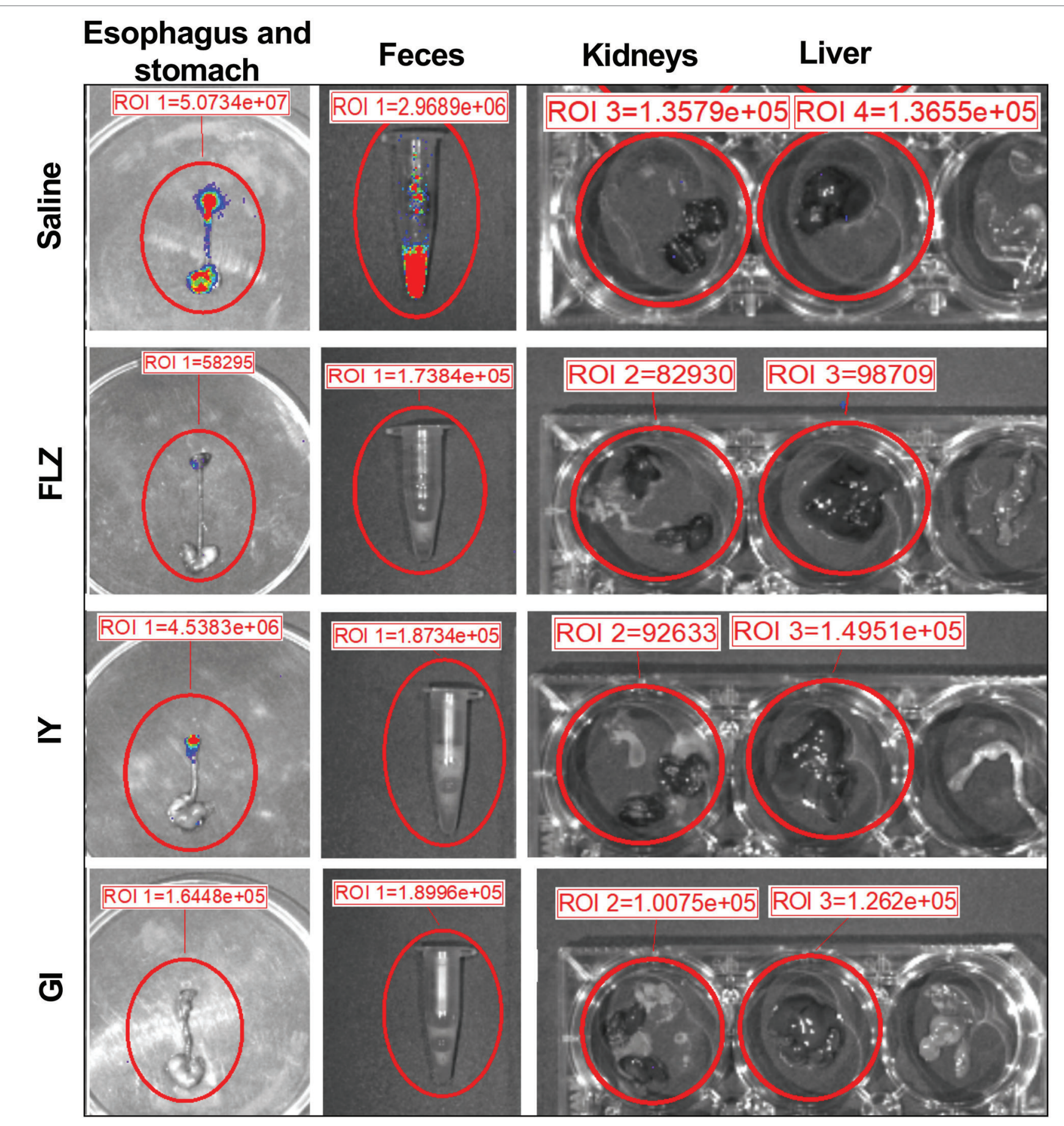

FIGURE 4 | Ex vivo imaging of mice orally infected with BLI-C. albicans and treated with FLZ, IY, or GI. Ex vivo analysis of esophagus, stomach, feces, kidneys and liver from saline, FLZ, IY or Gl-treated infected mice are shown. Briefly, 8 days post-infection mice were euthanized, and esophagus, stomach, kidneys, liver and feces were recovered and then soaked with $10 \mu \mathrm{l}$ of coelenterazine $(0.5 \mathrm{mg} / \mathrm{ml})$ to detect the $C$. albicans burden using the Lumina XRMS Imaging system. Data are from one representative mouse from one experiment. Region Of Interest (ROI) was quantified with Living Image $R$ software package.

process in the tongue. This effect was also observed when inactivated yeast was used instead of live yeast. Given that $S$. cerevisiae and C. albicans share some adhesins, it is likely that $S$. cerevisiae compete with the attachment of $C$. albicans
(Tada et al., 2002) to epithelial cells, thus inhibiting the colonization and dissemination of the pathogen. This can account for the complete resolution of the inflammatory process in the tongue of GI- and IY-treated infected mice. 

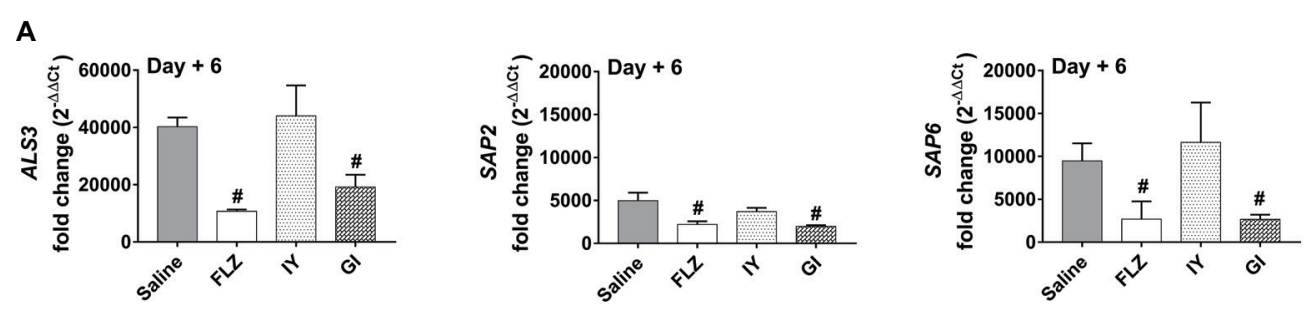

B
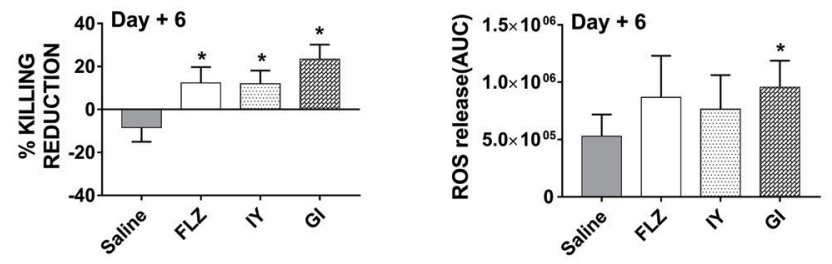

FIGURE 5 | Effect of IY or Gl on C. albicans virulence factors and on neutrophils activity. (A) ALS3, SAP2 and SAP6 gene expression in cellular fractions from tongue homogenates of infected mice treated with $10 \mu \mathrm{l}$ of saline, FLZ (4 mg/ml), IY, or Gl (both at $100 \mathrm{mg} / \mathrm{ml}$ ) at 1, 2, and 3 days post-infection was evaluated after 6 days of infection. Tongue homogenates were centrifuged, cellular fractions were lysed, and total RNA was extracted and retro-transcribed into cDNA. ALS3, SAP2, and SAP6 genes were detected as described in section "Materials and Methods." Data for ALS3, SAP2, and SAP6 genes are the mean \pm SEM from one experiment with $n=3$ mice/group. " $p<0.05$ FLZ or compound-treated infected mice vs. saline-treated infected mice. (B) Candidacidal activity of peritoneal neutrophils from infected mice, treated as above described, was evaluated after 6 days of infection. Data are the mean \pm SEM from two different experiments each with $n=3$ mice/group. ${ }^{*} p<0.05 \mathrm{FLZ}$ or compound-treated infected mice vs. saline-treated infected mice. Reactive oxygen species (ROS) of the peritoneal neutrophils $\left(4 \times 10^{6} / \mathrm{ml}\right)$ were evaluated after 6 days of infection in the presence of $C$. albicans $\left(2 \times 10^{6} \mathrm{CFU} / \mathrm{ml}\right)$. Quantification of total ROS production was determined by calculation of area under curve (AUC). Data are the mean \pm SEM from one experiment with $n=4$ mice/group. ${ }^{*} p<0.05 \mathrm{FLZ}$ or compound-treated infected mice vs. saline-treated infected mice.

C. albicans from the oral cavity (Hisajima et al., 2008; Patil et al., 2015) can spread to the pharynx and/or esophagus, stomach, and intestine (Mosci et al., 2013). In our experimental model, C. albicans cells were detected in the esophagus, stomach, and duodenum. The fungal load was progressively reduced after treatment with GI to the same extent as that observed with the conventional antifungal treatment (FLZ). In particular, no significant effect from GI and IY treatment was observed early on the infection time course (up to day +3 post-infection), while the beneficial effect of GI and IY was clearly noted later (from day +8 post-infection onward).

Late after the infection (on day +8 post-infection), C. albicans was recovered from the feces, suggesting the passage of Candida from the stomach to the gut without treatment (Mosci et al., 2013; Prieto and Pla, 2015). It is noteworthy that no such presence was observed in the mice treated with GI, IY, and FLZ, suggesting that the consistent reduction of $C$. albicans growth in various organs can prevent the intestine from being colonized/invaded by the pathogen. This is also relevant considering that the presence of $C$. albicans in the intestine is associated with different types of pathological conditions (Standaert-Vitse et al., 2009; Sokol et al., 2017).

Indeed, GI is able to reduce the expression of adhesion molecules such as ALS3 (Liu and Filler, 2011), and this is consistent with previously observed inhibition of the pathogen adhesion to epithelial cells (Pericolini et al., 2017; Gabrielli et al., 2018). In addition, GI treatment resulted in an impairment of the transition of C. albicans to mycelial form as well as of the expression of aspartyl proteases SAP2 and SAP6, both of which are highly involved in the colonization and dissemination of C. albicans (Naglik et al., 2003, 2004). In contrast to GI, IY is ineffective in regulating the expression of $C$. albicans virulence factors and yeast-to-hyphae transition. This may account for the lower protective effect obtained with IY.

Additional evidence of GI mediated effects is provided through the increased antimicrobial capacity of neutrophils related to an increase of oxygen-mediated mechanisms suggesting ROS-driven candidacidal activity.

Collectively, our results show that live and inactivated S. cerevisiae CNCM I-3856 is able to strongly reduce the local fungal burden commonly observed in OPC in the oral cavity, the esophagus, and the stomach, thus preventing the translocation of $C$. albicans to the small intestine. The inactivated yeast showed an inferior protective effect as compared to the live probiotic yeast. Furthermore, L. rhamnosus GG was completely ineffective. These results suggest that probiotic S. cerevisiae CNCM I-3856 is able to positively reverse/attenuate the course of OPC infection.

\section{DATA AVAILABILITY}

The raw data supporting the conclusions of this manuscript will be made available by the authors, without under reservation, to any qualified researcher.

\section{ETHICS STATEMENT}

All animal experiments were performed in agreement with the EU Directive 2010/63, the European Convention for the 
Protection of Vertebrate Animals used for Experimental and other Scientific Purposes, and the National Law 116/92. The protocol was approved by the Perugia University Ethics Committee and by the Modena and Reggio Emilia University Ethics Committee for animal care and use (Comitato Universitario di Bioetica and Organismo Preposto al Benessere degli Animali, permit number 223/2016-PR). The animals used for the realtime monitoring of oropharyngeal candidiasis were housed in the animal facility of the University of Modena and Reggio Emilia (Centro Servizi Stabulario Interdipartimentale, BIOSTAB, Authorization number 268/2011-A), and the animals used for all the other experiments were housed in the animal facility of the University of Perugia (Authorization number 34/2003A).

\section{AUTHOR CONTRIBUTIONS}

$\mathrm{AV}, \mathrm{NB}$, and $\mathrm{CM}$ conceived the study. AV, CM, and NB developed the project and designed the research. ER, SS, PM,

\section{REFERENCES}

Ai, R., Wei, J., Ma, D., Jiang, L., Dan, H., Zhou, Y., et al. (2017). A metaanalysis of randomized trials assessing the effects of probiotic preparations on oral candidiasis in the elderly. Arch. Oral Biol. 83, 187-192. doi: 10.1016/j. archoralbio.2017.04.030

Akpan, A., and Morgan, R. (2002). Oral candidiasis. Postgrad. Med. J. 78, 455-459. doi: 10.1136/pmj.78.922.455

Allonsius, C. N., van den Broek, M. F. L., De Boeck, I., Kiekens, S., Oerlemans, E. F. M., Kiekens, F., et al. (2017). Interplay between Lactobacillus rhamnosus GG and Candida and the involvement of exopolysaccharides. Microb. Biotechnol. 10, 1753-1763. doi: 10.1111/1751-7915.12799

Berberi, A., Noujeim, Z., and Aoun, G. (2015). Epidemiology of oropharyngeal candidiasis in human immunodeficiency virus/acquired immune deficiency syndrome patients and CD4+ counts. J. Int. Oral Health 7, 20-23.

Brummer, E., and Stevens, D. A. (1996). Synergy of human neutrophils with fluconazole in killing Candida species. Mycopathologia 134, 115-120. doi: 10.1007/BF00436717

Cagetti, M. G., Mastroberardino, S., Milia, E., Cocco, F., Lingstrom, P., and Campus, G. (2013). The use of probiotic strains in caries prevention: a systematic review. Nutrients 5, 2530-2550. doi: 10.3390/nu5072530

Enjalbert, B., Rachini, A., Vediyappan, G., Pietrella, D., Spaccapelo, R., Vecchiarelli, A., et al. (2009). A multifunctional, synthetic Gaussia princeps luciferase reporter for live imaging of Candida albicans infections. Infect. Immun. 77, 4847-4858. doi: 10.1128/IAI.00223-09

Ermert, D., Urban, C. F., Laube, B., Goosmann, C., Zychlinsky, A., and Brinkmann, V. (2009). Mouse neutrophil extracellular traps in microbial infections. J. Innate Immun. 1, 181-193. doi: 10.1159/000205281

European Committee for Standardization (2009). Animal feeding stuffs, CEN/TS 15790:2008. PCR typing of probiotic strains of Saccharomyces cerevisiae (yeast). ISBN 978-0-580-61806-2.

Food and Agriculture Organization and World Health Organization Expert Consultation (2001). Evaluation of health and nutritional properties of powder milk and live lactic acid bacteria. Córdoba, Argentina. Available from: ftp:// ftp.fao.org/es/esn/food/probio_report_en.pdf

Gabrielli, E., Pericolini, E., Ballet, N., Roselletti, E., Sabbatini, S., Mosci, P., et al. (2018). Saccharomyces cerevisiae-based probiotic as novel anti-fungal and anti-inflammatory agent for therapy of vaginal candidiasis. Benefic. Microbes 9, 219-230. doi: 10.3920/BM2017.0099

Garcia-Cuesta, C., Sarrion-Perez, M. G., and Bagan, J. V. (2014). Current treatment of oral candidiasis: a literature review. J. Clin. Exp. Dent. 6, e576-e582. doi: 10.4317/jced.51798 and $\mathrm{EP}$ performed the experiments. $\mathrm{AV}, \mathrm{CM}$, and $\mathrm{NB}$ wrote the manuscript. $\mathrm{EB}, \mathrm{SP}, \mathrm{AD}, \mathrm{AV}, \mathrm{CM}$, and $\mathrm{NB}$ analyzed and discussed the data. All authors contributed to the writing of the statement, agreed with its content and conclusions, and read and approved the final manuscript.

\section{FUNDING}

This work was done in the frame of a service agreement supported by Lesaffre International.

\section{ACKNOWLEDGMENTS}

The authors thank Sebastian Ku from Lesaffre Singapore for English language proofreading. We thank Dr. Daniela Fruttini, Department of Medicine, University of Perugia, for statistical assistance.

Gazendam, R. P., van de Geer, A., Roos, D., van den Berg, T. K., and Kuijpers T. W. (2016). How neutrophils kill fungi. Immunol. Rev. 273, 299-311. doi: 10.1111/imr.12454

Gonzalez Gravina, H., Gonzalez de Moran, E., Zambrano, O., Lozano Chourio, M., Rodriguez de Valero, S., Robertis, S., et al. (2007). Oral candidiasis in children and adolescents with cancer. Identification of Candida spp. Med. Oral Patol. Oral Cir. Bucal. 12, E419-E423

Gruner, D., Paris, S., and Schwendicke, F. (2016). Probiotics for managing caries and periodontitis: systematic review and meta-analysis. J. Dent. 48, 16-25. doi: 10.1016/j.jdent.2016.03.002

Gudlaugsson, O., Gillespie, S., Lee, K., Vande Berg, J., Hu, J., Messer, S., et al. (2003). Attributable mortality of nosocomial candidemia, revisited. Clin. Infect. Dis. 37, 1172-1177. doi: 10.1086/378745

Hatakka, K., Ahola, A. J., Yli-Knuuttila, H., Richardson, M., Poussa, T., Meurman, J. H., et al. (2007). Probiotics reduce the prevalence of oral Candida in the elderly - a randomized controlled trial. J. Dent. Res. 86, 125-130. doi: 10.1177/154405910708600204

Hisajima, T., Ishibashi, H., Yamada, T., Nishiyama, Y., Yamaguchi, H., Funakoshi, K., et al. (2008). Invasion process of Candida albicans to tongue surface in early stages of experimental murine oral candidiasis. Med. Mycol. 46, 697-704. doi: 10.1080/13693780802039919

Ishijima, S. A., Hayama, K., Burton, J. P., Reid, G., Okada, M., Matsushita, Y., et al. (2012). Effect of Streptococcus salivarius K12 on the in vitro growth of Candida albicans and its protective effect in an oral candidiasis model. Appl. Environ. Microbiol. 78, 2190-2199. doi: 10.1128/AEM.07055-11

Ishikawa, K. H., Mayer, M. P., Miyazima, T. Y., Matsubara, V. H., Silva, E. G., Paula, C. R., et al. (2015). A multispecies probiotic reduces oral Candida colonization in denture wearers. J. Prosthodont. 24, 194-199. doi: 10.1111/ jopr. 12198

Kenno, S., Perito, S., Mosci, P., Vecchiarelli, A., and Monari, C. (2016). Autophagy and reactive oxygen species are involved in neutrophil extracellular traps release induced by C. albicans morphotypes. Front. Microbiol. 7:879. doi: $10.3389 /$ fmicb.2016.00879

Kraft-Bodi, E., Jorgensen, M. R., Keller, M. K., Kragelund, C., and Twetman, S. (2015). Effect of probiotic bacteria on oral candida in frail elderly. J. Dent. Res. 94, 181S-186S. doi: 10.1177/0022034515595950

Kurtzman, C. P., and Robnett, C. J. (1997). Identification of clinically important ascomycetous yeasts based on nucleotide divergence in the $5^{\prime}$ end of the large-subunit (26S) ribosomal DNA gene. J. Clin. Microbiol. 35, 1216-1223.

Kurtzman, C. P., and Robnett, C. J. (1998). Identification and phylogeny of ascomycetous yeasts from analysis of nuclear large subunit (26S) ribosomal DNA partial sequences. Antonie Van Leeuwenhoek 73, 331-371. doi: 10.1023/A:1001761008817 
Lahtinen, S. J. (2012). Probiotic viability - does it matter? Microb. Ecol. Health Dis. 23(Suppl. 2). doi: 10.3402/mehd.v23i0.18567

Laleman, I., Detailleur, V., Slot, D. E., Slomka, V., Quirynen, M., and Teughels, W. (2014). Probiotics reduce mutans streptococci counts in humans: a systematic review and meta-analysis. Clin. Oral Investig. 18, 1539-1552. doi: 10.1007/ s00784-014-1228-z

Leão, M. V. P., Tavares, T. A. A., Goncalves, E. S. C. R., Dos Santos, S. S. F., Junqueira, J. C., de Oliveira, L. D., et al. (2018). Lactobacillus rhamnosus intake can prevent the development of candidiasis. Clin. Oral Investig. 22, 2511-2518. doi: 10.1007/s00784-018-2347-8

Li, X., Kolltveit, K. M., Tronstad, L., and Olsen, I. (2000). Systemic diseases caused by oral infection. Clin. Microbiol. Rev. 13, 547-558. doi: 10.1128/ CMR.13.4.547

Liu, Y., and Filler, S. G. (2011). Candida albicans ALS3, a multifunctional adhesin and invasin. Eukaryot. Cell 10, 168-173. doi: 10.1128/EC.00279-10

Matsubara, V. H., Bandara, H. M., Mayer, M. P., and Samaranayake, L. P. (2016). Probiotics as antifungals in mucosal candidiasis. Clin. Infect. Dis. 62, 1143-1153. doi: 10.1093/cid/ciw038

Melkoumov, A., Goupil, M., Louhichi, F., Raymond, M., de Repentigny, L., and Leclair, G. (2013). Nystatin nanosizing enhances in vitro and in vivo antifungal activity against Candida albicans. J. Antimicrob. Chemother. 68, 2099-2105. doi: 10.1093/jac/dkt137

Miranda, L. N., van der Heijden, I. M., Costa, S. F., Sousa, A. P., Sienra, R. A., Gobara, S., et al. (2009). Candida colonisation as a source for candidaemia. J. Hosp. Infect. 72, 9-16. doi: 10.1016/j.jhin.2009.02.009

Mosci, P., Gabrielli, E., Luciano, E., Perito, S., Cassone, A., Pericolini, E., et al. (2014). Involvement of IL-17A in preventing the development of deep-seated candidiasis from oropharyngeal infection. Microbes Infect. 16, 678-689. doi: 10.1016/j.micinf.2014.06.007

Mosci, P., Pericolini, E., Gabrielli, E., Kenno, S., Perito, S., Bistoni, F., et al. (2013). A novel bioluminescence mouse model for monitoring oropharyngeal candidiasis in mice. Virulence 4, 250-254. doi: 10.4161/viru.23529

Moyes, D. L., and Naglik, J. R. (2011). Mucosal immunity and Candida albicans infection. Clin. Dev. Immunol. 2011:346307. doi: 10.1155/2011/346307

Naglik, J., Albrecht, A., Bader, O., and Hube, B. (2004). Candida albicans proteinases and host/pathogen interactions. Cell. Microbiol. 6, 915-926. doi: 10.1111/j.1462-5822.2004.00439.x

Naglik, J. R., Challacombe, S. J., and Hube, B. (2003). Candida albicans secreted aspartyl proteinases in virulence and pathogenesis. Microbiol. Mol. Biol. Rev. 67, 400-428. doi: 10.1128/MMBR.67.3.400-428.2003

Naglik, J. R., and Moyes, D. (2011). Epithelial cell innate response to Candida albicans. Adv. Dent. Res. 23, 50-55. doi: 10.1177/0022034511399285

Naglik, J. R., Moyes, D., Makwana, J., Kanzaria, P., Tsichlaki, E., Weindl, G., et al. (2008). Quantitative expression of the Candida albicans secreted aspartyl proteinase gene family in human oral and vaginal candidiasis. Microbiology 154, 3266-3280. doi: 10.1099/mic.0.2008/022293-0

Naglik, J. R., Moyes, D. L., Wachtler, B., and Hube, B. (2011). Candida albicans interactions with epithelial cells and mucosal immunity. Microbes Infect. 13, 963-976. doi: 10.1016/j.micinf.2011.06.009

Ohshima, T., Kojima, Y., Seneviratne, C. J., and Maeda, N. (2016). Therapeutic application of synbiotics, a fusion of probiotics and prebiotics, and biogenics as a new concept for oral Candida infections: a mini review. Front. Microbiol. 7:10. doi: $10.3389 /$ fmicb.2016.00010

Pappas, P. G. (2006). Invasive candidiasis. Infect. Dis. Clin. N. Am. 2, 485-506. doi: 10.1016/j.idc.2006.07.004

Patil, S., Rao, R. S., Majumdar, B., and Anil, S. (2015). Clinical appearance of oral Candida infection and therapeutic strategies. Front. Microbiol. 6:1391. doi: $10.3389 /$ fmicb. 2015.01391
Pericolini, E., Gabrielli, E., Ballet, N., Sabbatini, S., Roselletti, E., Cayzeele Decherf, A., et al. (2017). Therapeutic activity of a Saccharomyces cerevisiaebased probiotic and inactivated whole yeast on vaginal candidiasis. Virulence 8, 74-90. doi: 10.1080/21505594.2016.1213937

Premanathan, M., Shakurfow, F. A. A., Ismail, A. A., Berfad, M. A., Ebrahim, A. T., and Awaj, M. M. (2011). Treatment of oral candidiasis (thrush) by Saccharomyces cerevisiae. Int. J. Med. Med. Sci. 3, 83-86.

Prieto, D., and Pla, J. (2015). Distinct stages during colonization of the mouse gastrointestinal tract by Candida albicans. Front. Microbiol. 6:792. doi: 10.3389/ fmicb.2015.00792

Ricci, E., Ronchetti, S., Gabrielli, E., Pericolini, E., Gentili, M., Roselletti, E., et al. (2019). GILZ restrains neutrophil activation by inhibiting the MAPK pathway. J. Leukoc. Biol. 105, 187-194. doi: 10.1002/JLB.3AB0718-255R

Roudbarmohammadi, S., Roudbary, M., Bakhshi, B., Katiraee, F., Mohammadi, R., and Falahati, M. (2016). ALS1 and ALS3 gene expression and biofilm formation in Candida albicans isolated from vulvovaginal candidiasis. $A d v$. Biomed. Res. 5:105. doi: 10.4103/2277-9175.183666

Sabbatini, S., Monari, C., Ballet, N., Mosci, P., Cayzeele Decherf, A., Pélerin, F., et al. (2018). Saccharomyces cerevisiae-based probiotic as novel anti-microbial agent for therapy of bacterial vaginosis. Virulence 9, 954-966. doi: 10.1080/21505594.2018.1464362

Sardi, J. C., Scorzoni, L., Bernardi, T., Fusco-Almeida, A. M., and Mendes Giannini, M. J. (2013). Candida species: current epidemiology, pathogenicity, biofilm formation, natural antifungal products and new therapeutic options. J. Med. Microbiol. 62, 10-24. doi: 10.1099/jmm.0.045054-0

Schulze, J., and Sonnenborn, U. (2009). Yeasts in the gut: from commensals to infectious agents. Dtsch. Arztebl. Int. 106, 837-842. doi: 10.3238/arztebl.2009.0837

Sokol, H., Leducq, V., Aschard, H., Pham, H. P., Jegou, S., Landman, C., et al. (2017). Fungal microbiota dysbiosis in IBD. Gut 66, 1039-1048. doi: 10.1136/ gutjnl-2015-310746

Solis, N. V., and Filler, S. G. (2012). Mouse model of oropharyngeal candidiasis. Nat. Protoc. 7, 637-642. doi: 10.1038/nprot.2012.011

Standaert-Vitse, A., Sendid, B., Joossens, M., Francois, N., Vandewalle-El Khoury, P., Branche, J., et al. (2009). Candida albicans colonization and ASCA in familial Crohn's disease. Am. J. Gastroenterol. 104, 1745-1753. doi: 10.1038/ajg.2009.225

Tada, H., Nemoto, E., Shimauchi, H., Watanabe, T., Mikami, T., Matsumoto, T., et al. (2002). Saccharomyces cerevisiae- and Candida albicans-derived mannan induced production of tumor necrosis factor alpha by human monocytes in a CD14- and toll-like receptor 4-dependent manner. Microbiol. Immunol. 46, 503-512. doi: 10.1111/j.1348-0421.2002.tb02727.x

Weindl, G., Naglik, J. R., Kaesler, S., Biedermann, T., Hube, B., Korting, H. C., et al. (2007). Human epithelial cells establish direct antifungal defense through TLR4-mediated signaling. J. Clin. Invest. 117, 3664-3672. doi: 10.1172/JCI28115

Conflict of Interest Statement: $\mathrm{NB}$ and $\mathrm{AD}$ were employed by the company Lesaffre International, Lesaffre Group, Marcq-en-Baroeul, France.

The remaining authors declare that the research was conducted in the absence of any commercial or financial relationships that could be construed as a potential conflict of interest.

Copyright (c) 2019 Roselletti, Sabbatini, Ballet, Perito, Pericolini, Blasi, Mosci, Cayzeele Decherf, Monari and Vecchiarelli. This is an open-access article distributed under the terms of the Creative Commons Attribution License (CC BY). The use, distribution or reproduction in other forums is permitted, provided the original author(s) and the copyright owner(s) are credited and that the original publication in this journal is cited, in accordance with accepted academic practice. No use, distribution or reproduction is permitted which does not comply with these terms. 